\title{
Program Development in the Constructive Set Theory TK
}

\author{
Martin C. Henson \\ Department of Computer Science, University of Essex, Wivenhoe Park, Colchester, Essex, \\ United Kingdom
}

Keywords: Constructive set theory; Program development; Specification; Transformation; Types; Verification

\begin{abstract}
We present a constructive theory of types and kinds designed with program development as the major desideratum. We show how this theory may be employed to derive programs from proofs of specifications (that is, demonstrations that specifications are satisfiable) and how the infrastructure of the theory supports the transformational development of programs in a natural way.
\end{abstract}

\section{Introduction}

In this paper we investigate some aspects of program derivation and transformation within a constructive set theory known as TK. In recent years there has been a great deal of interest in the development of programs within some constructive framework. The most popular theory seems to be Martin Lof's constructive theory of types (MLTT) [Mar82] [Abb87] [Con86] [BCM89] [Kha86] although other paradigms have also been studied, for example, the Theory of Constructions [Moh85] and Feferman's theory $T_{0}$ [HaN87]. The theory TK was introduced in [HeT88] and there we devoted our attention to basic motivation and mathematical aspects. We have, however, endeavoured to keep the current paper self contained.

At this stage in the development of theories of this kind our aims are still quite modest: we are looking for organisational clarity, in particular, theories which do not force us to operate with unnatural program and type structures. This latter point is crucial: research in this area has demonstrated that the basic

Correspondence and offprint requests to: Martin C. Henson, Department of Computer Science, University of Essex, Wivenhoe Park, Colchester, Essex, UK. 
principles of constructive reasoning can be harnessed for rudimentary program development but, in particular, the language in which the programs are expressed, and the nature of the type structures themselves, do not necessarily conform to those which we have learned, from more practical research in computer science, are most appropriate. For example, in MLTT, the programs are rather low level objects (a typed lambda calculus) and the principle of unicity of types prevents the most common forms of type polymorphism. Much of the current research is aimed at addressing these topics. Many new type structures have been advocated for MLTT which help to ameliorate these problems. Two recent examples, concerning recursive and polymorphic types (respectively), are [Nor87] and [MaC88]. Our approach has been to design a theory with program development (rather than an account of constructive mathematics) as the major desideratum. We feel that, in the long run, this will prove a more systematic approach and will lead ultimately to a more coherent framework. We also feel that research into the design of theories of (and for) program development is very much in its infancy and most of the current work essentially foundational in nature. Consequently in reading this, or any paper in this area, the reader should not expect to see, at least at this stage of development, fully fledged systems for software derivation but deliberations at a rather more fundamental level, concerning the basic formalisation on which we hope in the future such useful systems may be developed.

As a consequence of our aims the examples of Sections 3 and 4 are quite modest and we use them to illustrate certain general principles, to provide guidance for further development of the underlying theory, and to contrast the theory TK with other frameworks which have been used for program development. Section 3 concerns the extraction of programs from the proofs of their specifications, that is, from the proofs that specifications are in principle satisfiable. This is quite a familiar enterprise to readers conversant with MLTT although we shall consider a case which involves general recursion, a topic which has only been studied a little in MLTT. Our aim is to show that (terminating) general recursion can be handled in TK in a natural way. The claim to naturalness here is based, as we shall see, on a connection between recursive structures and abstract data types. In Section 4 we turn to transformational programming. We will investigate some fairly well known examples in order to demonstrate how transformation can be understood within an intensional theory of sets. The examples are again quite modest but have been deliberately chosen to be illustrative of the principles of intensionality and flexible typing which seem to provide a natural account of transformation in the theory we are presenting.

\section{The Constructive Set Theory TK}

In this section we introduce briefly the set theory TK. In Section 2.1 we outline the language of the theory and, in Section 2.2, the system of rules.

\subsection{The Language of the Theory TK}

The theory consists of a language of terms, sets and formulae. We employ the following syntactic categories and variables to range over them. In brackets we indicate (very roughly) the role each category plays in the practice of program development. 


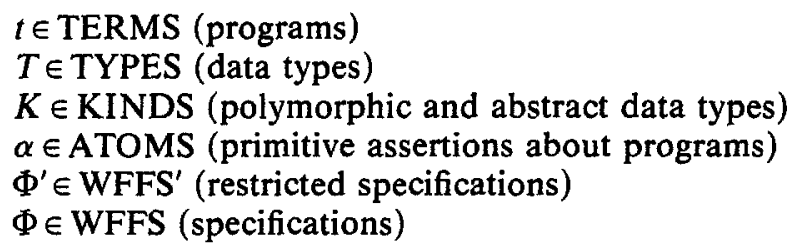

The term language consists of the untyped lambda notation extended with constants, abstractions over the types, and types as objects:

$$
t \rightarrow x|c| \lambda x . t|\lambda X . T|(t t) \mid T
$$

We will use, without further elaboration, standard syntactic sugarings for pairing and selectors, conditional, boolean constants and so on. Other sugarings are more sophisticated and in a sense constitute a formal compilation process: we write, in particular, function definitions using pattern directed recursion equations. These are unpacked via a collection of combinators (which are of course lambda definable) due to Turner [Tur79] (but see also [Hen87] or [Pey87] for example).

Sets are called types or kinds. Although both classify objects of the theory the kinds also classify those objects which are constructed from types.

$$
\begin{aligned}
& T \rightarrow X\left|\left\{x \mid \Phi^{\prime}\right\}\right| \Xi(\Phi, T) \\
& K \rightarrow T \mid\left\{x \mid \Phi^{\prime}\right\}
\end{aligned}
$$

Sets can be formed from properties by comprehension and by induction. Comprehension, we will see, supports the introduction of well known type constructions, such as sums, products, function spaces, polymorphisms and data abstractions. Inductive generation allows us to satisfy certain recursive type definitions, some of which we will see later. Intuitively, $\Xi(\Phi, T)$ is a set built up from a base set $T$ by means of a property $\Phi$. We will see this precisely in Section 2.2.

Atomic formulae consist of membership, equality, definedness and absurdity assertions:

$$
\alpha \rightarrow t \in T|t \in K| t=t|t \downarrow| \perp
$$

We can then close off the assertions in the normal way, although we include quantifiers over types as well as objects. We can extend quantification to kinds but have not done so here because we shall not need it.

$$
\Phi \rightarrow \alpha|\Phi \wedge \Phi| \Phi \vee \Phi|\Phi \Rightarrow \Phi|(\forall x) \Phi|(\exists x) \Phi|(\forall X) \Phi \mid(\exists X) \Phi
$$

The set WFF' over which $\Phi^{\prime}$ ranges is that subset of WFF which includes no element involving a bound type variable (by quantification).

This completes the presentation of the basic language of the theory. The presentation we have just given differs from that given in [HeT88] but only in the sense that it is a sub-theory which we have described here. Full TK consists of a hierarchy of kind levels, with quantification at each level. The inclusion of such quantification means that there are restrictions on the properties involved in comprehension types at each level in full TK. Here there are no restrictions at the kind level becuase we eschew kind quantification. Full TK is not necessary for the elaboration of the material we present in this paper; indeed we have attempted to isolate the weakest sub-theory of TK which can support the techniques we require in the sequel. 


\subsection{The Rule System of TK}

The logical apparatus of TK falls into two parts. First we have the intuitionistic logic of partial terms. This is a version of intuitionistic predicate calculus which allows the formation of terms which may not denote. Term and formulae formation remain as usual but atomic formulae are extended to include the assertion $t \downarrow$ which is to be read " $t$ denotes". The only changes to the inference rules concern the rules for universal elimination, existential introduction and equality. The former are recast in such a way as to remove the possibility of inferring something of a non-denoting term.

$$
(\forall-E \lim ) \frac{(\forall x) \Phi t \downarrow}{\Phi(t)} \quad(\exists-\text { Intro }) \frac{\Phi(t) t \downarrow}{(\exists t) \Phi}
$$

We assume a standard rule system for the rest of the logic but will not list it here. When it becomes necessary to be more explicit we will provide the rules we need.

For equality we introduce the shorthand $t \simeq t^{\prime}$ for $\left(t \downarrow \vee t^{\prime} \downarrow\right) \Rightarrow t=t^{\prime}$. The axioms for equality and definedness are then:

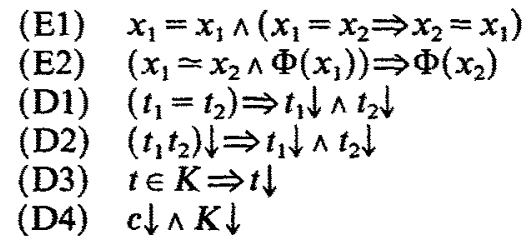

Beeson shows [Bee85] that this logic of partial terms is only a convenient expression for standard intuitionistic predicate calculus.

The axioms for the partial lambda calculus are now expressed as follows:

$$
\begin{array}{ll}
\text { (alpha) } & \lambda x . t=\lambda y \cdot t[x \leftarrow y](y \text { not free in } t) \\
\text { (beta) } & (\lambda x . t) y \simeq t[x \leftarrow y] \\
\text { (beta') } & (\lambda X . T) Y \approx T[X \leftarrow Y]
\end{array}
$$

The other expected rules for the lambda calculus now follow as elementary consequences of axiom (E2).

We can now turn to the second category, the rules for introduction and elimination of membership in sets:

$$
\begin{gathered}
\frac{z \in\{x \mid \Phi\}}{\Phi(z)}(\{\} \text {-Elim }) \frac{\Phi(z)}{z \in\{x \mid \Phi\}}(\{\} \text {-Intro }) \\
\frac{z \in T}{z \in \Xi(\Phi, T)}(\Xi-\operatorname{Intro}(i)) \frac{(\forall x)(\Phi(z, x) \Rightarrow x \in \Xi(\Phi, T))}{z \in \Xi(\Phi, T)}(\Xi-I n t r o(i i)) \\
\frac{(\forall z \in T) \psi(z)(\forall z \in \operatorname{Clo}(\Xi(\Phi, T)))((\forall x \in\{v \in \Xi(\Phi, T) \mid \Phi(z, v)\})(\psi(x)) \Rightarrow \psi(z))}{(\forall z \in \Xi(\Phi, T)) \psi(z)} \\
(\Xi-\operatorname{Elim})
\end{gathered}
$$

where $\operatorname{Clo}(\Xi(\Phi, T))$ is $\{z \mid(\forall x)(\Phi(z, x) \Rightarrow x \in \Xi(\Phi, T))\}$.

Although there is no logical distinction to make between bounded quantification of the form $(\forall z \in T) \psi(z)$ used in the rule above, and the unbounded version: $(\forall z)(z \in T \Rightarrow \psi(z))$ we shall see in Section 2.3 that the interpretations 
of these two formulae (via realizability) can, in certain precise circumstances, be distinct. It is, we shall see, a feature of TK that formulae which are logically equivalent but intensionally distinct (here this means just that they have different presentations) lead to distinct interpretations.

Another point worth making here concerns the sets of TK. These inherit the equality relation because sets are included as terms. However, there are no special rules for set equality. In particular, there is no rule which corresponds to extensionality in classical set theory. In fact the theory TK + ext is inconsistent. Sets are only equal if they are identical. This makes TK an intensional set theory. Other useful relations on sets (including, of course, a notion of extensional equality) can be defined within the theory and, indeed, we shall do this later. We shall, in the sequel, discuss the importance of intensionality for theories of program development.

\subsection{Realizability and Program Extraction}

Unlike MLTT, our proof rules are about formulae and not types. It is necessary to extract the constructive information fro $n$ proofs by some other means. The mechanism we use is a modified realizability interpr station. One of the reasons we have split the activities of proving and program extraction is that it provides us with the opportunity of investigating a variety of possible program extraction frameworks, without having to modify the underlying theory. We firmly believe that the design and investigation of theories of program development is a topic very much in its infancy; we do not think it is by any means clear what all the issues are and we are loathe to commit ourselves to any fixed system for the present.

In this section we introduce a notion of realizability for TK and show how it can be used to obtain programs (in the sugared term language) from proofs of specifications (articulated in the assertion language). The technique of realizability was originally introduced [Kle45] to capture formally the constructive meaning of the logical operations. To each formula, $\Phi$, we associate another, written " $e \rho \Phi$ " which is to be read " $e$ realizes $\Phi$ " or more suggestively: " $e$ is a program which meets the specification $\Phi$ ". The idea is that the translation makes the constructive character of assertions explicit.

Before we move on, we need to make a few technical remarks. If the free variables of $\Phi$ are $\bar{x}$ and $\bar{X}$, then those of $e \rho \Phi$ are $\bar{x}, \bar{X}, \bar{X}^{*}$ and $e$, where $e$ is a fresh object variable and the $\bar{X}^{*}$ are fresh type variables, each of which is associated (in a manner described in Lemma 2.3.3) to a type variable in $\bar{X}$. We shall take it that $\rho$ binds more tightly in expressions than the binary logical connectives but less tightly than quantifiers and membership (note, however, that expressions of the form $e \rho \Phi$ are meta-notation). We now define, by mutual induction two concepts: the negative formulae and the negative sets, as follows.

Definition 2.3.1

The predicates $\operatorname{neg}(\Phi)$ ( $\Phi$ is negative) and $\operatorname{neg}(T)$ ( $T$ is negative) are defined as follows:

$$
\begin{aligned}
& \operatorname{neg}(\perp) \\
& \operatorname{neg}(t \downarrow) \\
& \operatorname{neg}\left(t=t^{\prime}\right)
\end{aligned}
$$




$$
\begin{aligned}
& \operatorname{neg}(T) \Rightarrow \operatorname{neg}(t \in T) \\
& \operatorname{neg}(\Phi) \wedge \operatorname{neg}(\psi) \Rightarrow \operatorname{neg}(\Phi \wedge \psi) \\
& \operatorname{neg}(\psi) \Rightarrow \operatorname{neg}(\Phi \Rightarrow \psi) \\
& \operatorname{neg}(\Phi) \Rightarrow \operatorname{neg}((\forall x) \Phi) \\
& \operatorname{neg}(\Phi) \Rightarrow \operatorname{neg}(\{x \mid \Phi\}) \\
& \operatorname{neg}(\Phi) \wedge \operatorname{neg}(T) \Rightarrow \operatorname{neg}(\Xi(\Phi, T))
\end{aligned}
$$

\section{Definition 2.3.2}

$$
\begin{aligned}
& \text { e } \rho \Phi \quad \text { is } \Phi(\mathrm{neg}(\Phi)) \\
& e \rho t \in X \quad \text { is }\langle e, t\rangle \in X^{*} \\
& e \rho t \in\{x \mid \Phi\} \quad \text { is }\langle e, t\rangle \in\{x \mid \Phi\}^{*} \\
& \text { e } \rho t \in \Xi(\Phi, T) \quad \text { is }\langle e, t\rangle \in \Xi(\Phi, T)^{*} \\
& e \rho(\Phi \wedge \psi) \quad \text { is } \Phi \wedge e \rho \psi(\operatorname{neg}(\Phi)) \\
& e \rho(\Phi \wedge \psi) \quad \text { is } e \rho \Phi \wedge \psi(\operatorname{neg}(\psi)) \\
& e \rho(\Phi \wedge \psi) \quad \text { is (fst } e) \rho \Phi \wedge \text { (snd } e) \rho \psi \text { (otherwise) } \\
& e \rho(t \rightarrow \Phi, \psi) \quad \text { is } t \rightarrow e \rho \Phi, e \rho \psi \\
& e \rho(\Phi \vee \psi) \quad \text { is (fst } e) \rightarrow \Phi \wedge \text { (snd } e) \rho \Phi, \psi \wedge \text { (snd } e) \rho \psi \\
& e \rho(\Phi \Rightarrow \psi) \quad \text { is }(\forall x)(\Phi \wedge x \rho \Phi \Rightarrow(e x) \downarrow \wedge(e x) \rho \psi) \\
& e \rho(\forall x \in T) \Phi \quad \text { is }(\forall x \in T)((e x) \rho \Phi)(\text { for, at most, } \operatorname{neg}(T)) \\
& e \rho(\forall x) \Phi \quad \text { is }(\forall x)((e x) \downarrow \wedge(e x) \rho \Phi) \\
& e \rho \text { let } p=x \text { in } \Phi \text { is let } p=x \text { in }(e \rho \Phi) \\
& e \rho(\exists x) \Phi \quad \text { is } \Phi((\text { fst } e)) \wedge(\text { snd } e) \rho \Phi((\text { fst } e)) \\
& e \rho(\forall X) \Phi \quad \text { is }(\forall X)(e \rho \Phi) \\
& e \rho(\exists X) \Phi \quad \text { is }(\exists X)(X=(\mathrm{fst} e) \wedge \Phi \wedge(\text { snd } e) \rho \Phi) \\
& \text { e } \rho \neg \Phi \quad \text { is } e \rho(\Phi \Rightarrow \perp) \\
& \text { e } \rho(\Phi \Leftrightarrow \psi) \quad \text { is } \Phi \Rightarrow \psi \wedge \psi \Rightarrow \Phi
\end{aligned}
$$

The detailed definitions of the types $T^{*}$, used in the definition above, need not concern us in the present paper and we shall not need to refer to them again. However, the following lemma is a crucial component of the technical results which follow.

\section{Lemma 2.3.3}

$$
e \rho(\Phi(\bar{x}, \bar{X})[\bar{X} \leftarrow \bar{T}])=\left((e \rho \Phi)\left(\bar{X}, \bar{X}, \bar{X}^{*}\right)\right)[\bar{X} \leftarrow \bar{T}]\left[\bar{X}^{*} \leftarrow \bar{T}^{*}\right]
$$

There are one or two non-standard assertion forms countenanced by Definition 2.3.2. All are, of course, just syntactic sugar for standard assertions. Including them explicitly in the definition is, however, quite important because realizability interpretations are very intensional. That is, they depend not only on the logical force of assertions but also on the way in which they are presented to us. For example consider two distinct, non-negative formulae $\Phi$ and $\psi$; then obviously: $\Phi \wedge \psi \Leftrightarrow \psi \wedge \Phi$ but a realizer of $\Phi \wedge \psi$ is not a realizer of $\psi \wedge \Phi$. Of course there is a relationship between the realizers: if $\Phi \Leftrightarrow \psi$ (for any formulae $\Phi$ and $\psi$ ) then by soundness (Proposition 2.3.4) there is a $t$ such that $t \rho(\Phi \Leftrightarrow \psi)$. If $e$ realizes $\Phi$ then (fst $t e$ ) realizes $\psi$ and in, in fact, we can define an extensional equality for terms (written $t \equiv t^{\prime}$ ) so that if $e^{\prime}$ realizes $\psi$ then (fst $t e$ ) $\equiv e^{\prime}$.

The simplest non-standard forms are the bounded universal quantifiers. In some contexts, we wish to restrict the use of these quantifiers to just a small collection of negative sets, in particular just a decidable fragment; this explains the side condition above. It is important to see that the interpretation they are 
given above is not that which they would otherwise inherit from the standard clauses for unbounded quantification and implication. The distinction amounts essentially to the difference between compile and run-time typing. Realizers of the bounded assertions correspond to the former, whereas those of the syntactic unpacking correspond to run-time typing. The conditional assertion and the let assertion are rather less familiar (although formulae of a similar, but by no means equivalent, form appear in [HaN87]). Conditional assertions may be unpacked in two equivalent ways:

$$
\begin{aligned}
& \left.t \rightarrow \Phi, \psi \Leftrightarrow \Leftrightarrow_{\text {def }}(t=\operatorname{true} \wedge \Phi) \vee(t=\text { false } \wedge \psi) \quad \text { ( } \vee \text {-presentation }\right) \\
& t \rightarrow \Phi, \psi \Leftrightarrow \Leftrightarrow_{\text {def }} t \in \operatorname{Bool} \wedge(t=\text { true } \Rightarrow \Phi) \wedge(t=\text { false } \Rightarrow \psi) \quad(\wedge \text {-presentation })
\end{aligned}
$$

These give rise to a number of derived rules for conditional assertion introduction and elimination which the reader is invited to construct. Note that realizers of conditional assertions are not realizers of either of the unpackings above. Another important point: suppose that $\Phi$ is decidable then there is a term $t$ so that: $t=$ true $\Leftrightarrow \Phi \wedge t=$ false $\Leftrightarrow \neg \Phi$. In such circumstances we shall sometimes use the conditional as follows: $\Phi \rightarrow \psi, \eta$.

Let assertions are a little more complicated. In the assertion: "let $p=x$ in $\Phi$ " we take $p$ to be a pattern in the sense this is used in a number of functional programming languages. That is to say, $p$ is an exprzssion built from variables and constants (where some of the constants may be data constructors). All variables in a pattern are free, or more exactly, there are no binding operators in patterns. We write $\operatorname{Var}(p)$ for the set of variables which occur in $p$. We can now unpack such expressions:

$$
\text { let } p=x \text { in } \Phi \Leftrightarrow_{\text {def }}\left(\exists x_{1}\right) \ldots\left(\exists x_{n}\right)(x=p \wedge \Phi)
$$

In this the existentially bound variables number all the elements of $\operatorname{Var}(p)$. Again, note that the realizers of such expressions are quite different from those which realize the unpackings. There are also derived rules for introducing and eliminating let-assertion formulae which are easy to construct. It is important to point out that there is an alternative and equivalent unpacking of let assertions:

$$
\begin{aligned}
& \text { let } p=x \text { in } \Phi \Leftrightarrow_{\text {der }} \neg \neg\left(\exists x_{1}\right) \cdots\left(\exists x_{n}\right)(x=p \wedge \Phi) \wedge \\
& \left(\forall x_{1}\right) \cdots\left(\forall x_{n}\right)(x=p \Rightarrow \Phi)
\end{aligned}
$$

The reader is referred to [HaN87] for further details. Note that there are negative formulations for both conditional and let assertion forms, consequently we can extend the class of negative formulae to include both $t \rightarrow \Phi, \psi$ and let $p=x$ in $\Phi$ when $\Phi$ and $\psi$ are negative.

We view realizability as expressing the meaning of the logical apparatus. Thus we can think of realizability as defining a model in the following sense: if $\Phi$ can be proved then there is a $t$ such that $t \rho \Phi$ (can be proved). More exactly, we have the following proposition which lies at the heart of the process of program extraction.

\section{Proposition 2.3.4 (soundness of realizability)}

If $\mathrm{TK} \vdash \Phi$ then there is a term $t$ such that $\mathrm{TK} \vdash t \downarrow \wedge t \rho \Phi$.

Proof sketch. The proof proceeds by induction on the length of the derivation TK $\vdash \Phi$. Essentially, this means that we have to construct a combinator for each proof rule which, given realizing terms of the premises, can construct a realizing 
term for the consequent. We provide two examples, the first of which concerns the rule for $\rightarrow$-elimination ( $\vee$-presentation). The derived rule is:

\begin{tabular}{ccc} 
& {$[t=\operatorname{true} \wedge \Phi]$} & {$[t=$ false $\wedge \psi]$} \\
$t \rightarrow \Phi, \psi$ & $\vdots$ & $\vdots$ \\
& $\eta$ & $\eta$ \\
\hline
\end{tabular}

Suppose, ex hypothesi, that $t_{1} \rho t \rightarrow \Phi, \psi$; that $t_{2}\left(z_{1}\right) \rho \eta$ whenever $z_{1} \rho t=$ true $\wedge \Phi$ and that $t_{3}\left(z_{2}\right) \rho \eta$ whenever $z_{2} \rho t=$ false $\wedge \psi$. By the definition of realizability for conditional assertions, we have: $t \rightarrow t_{1} \rho \Phi, t_{1} \rho \psi$. Note that if $z_{1} \rho t=$ true $\wedge \Phi$, then we have: $t=$ true $\wedge z_{1} \rho \Phi$, since $t=$ true is negative, and if $z_{2} \rho t=$ false $\wedge \psi$ then we have: $t=$ false $\wedge z_{2} \rho \psi$, since $t=$ false is negative. Now assume that $t=$ true $\wedge$ $t_{1} \rho \Phi$, so therefore $t_{2}\left(t_{1}\right) \rho \eta$. By $\rightarrow$-introduction ( $v$-presentation) we conclude that $t_{1} \rightarrow t_{2}\left(t_{1}\right), t_{3}\left(t_{1}\right) \rho \eta$. On the other hand, assume that $t=$ false $\wedge t_{1} \rho \psi$ then $t_{3}\left(t_{1}\right) \rho \eta$ and by $\rightarrow$-introduction we again see that $t \rightarrow t_{2}\left(t_{1}\right), t_{3}\left(t_{1}\right) \rho \eta$. Discharging the two assumptions we obtain, by $\rightarrow$-elimination ( $v$-presentation), that $t \rightarrow$ $t_{2}\left(t_{1}\right), t_{3}\left(t_{1}\right) \rho \eta$ is the realizer of the conclusion of the rule: $\rightarrow$-elimination $(v-$ presentation). The combinator in question is: $\lambda x . \lambda y \cdot \lambda z . t \rightarrow y(x), z(x)$. We have suppressed the argumentation that ensures the realizer is defined, but this is straightforward.

Our second example concerns the combinator associated with the rule for induction. We use the modified realizability for bounded quantification here and so the analysis is sound providing that $T$ is a negative set and $\Phi$ is a negative formula. The reader will note that the examples we work with later in this paper conform to this regime.

$(\forall z \in T) \psi(z)(\forall z \in \operatorname{Clo}(\Xi(\Phi, T)))((\forall x \in\{v \in \Xi(\Phi, T) \mid \Phi(z, v)\})(\psi(x)) \Rightarrow \psi(z))$

( $\Xi$-Elim)

$(\forall z \in \Xi(\Phi, T)) \psi(z)$

Suppose that $t_{1}$ and $t_{2}$ are defined and realize the two premises (ex hypothesi). We need to define a combinator, irec, so that (irec $t_{1} t_{2}$ ) realizes the conclusion. That is, for every $z$, (irec $t_{1} t_{2} z$ ) is defined and realizes $\psi(z)$. We proceed by $\Xi(\Phi, T)$-induction: If $z$ belongs to $T$ then $\left(t_{1} z\right)$ will realize $\psi(z)$. On the other hand, if $z$ belongs to $\operatorname{Clo}(\Xi(\Phi, T))$ then we can use $t_{2}$ as follows: $\left(t_{2} z w\right)$ realizes $\psi(z)$ if we can find a $w$ which realizes $(\forall x \in\{v \in \Xi(\Phi, T) \mid \Phi(z, v)\}) \psi(x)$. That is $(w y)$ realizes $\psi(x)$ whenever $y$ belongs to $\{v \in \Xi(\Phi, T) \mid \Phi(z, v)\}$. This is obtained (ex hypothesi) by taking $w$ to be $\lambda x$. (irec $t_{1} t_{2} x$ ). To summarise: irec has to satisfy:

irec $x y z=x z$

irec $x y z=y z(\lambda v$.(irec $x y v))$ (when $z \in T$ )

(otherwise)

Indeed, providing that membership in $T$ is decidable, we can take these equations as defining irec since, as we indicated in the introduction, we know how to unpack such pattern directed equations in our term language by standard compilation techniques. The general case (where $\Xi(\Phi, T)$ is constructed from arbitrary $T$ and $\Phi)$ can be accommodated by a somewhat more complex definition of irec.

We do not base our program extraction from proofs directly on realizability, but via a rule corresponding to the negative axiom of choice $A C_{\text {neg }}$ as in [Bee85]. Specifications are given in terms of a (negative) precondition $\Phi$ and a postcondition $\psi$ and they assert that there is a value which satisfies the postcondition for every value which satisfies the precondition. 


\section{Proposition 2.3.5. Program Extraction}

If $\mathrm{TK} \vdash(\forall x)(\Phi(x) \Rightarrow(\exists y)(\psi(x, y)))$ where the precondition $\Phi(x)$ is negative, then for some closed term $t$ :

$$
\operatorname{TK} \vdash(\forall x)(\Phi(x) \Rightarrow(t x) \downarrow \wedge \psi(x,(t x))) .
$$

Proof. is a simple consequence of Proposition 2.3.4.

Since specifications of programs may embed formulae of the kind considered in the proposition above (typically they are embedded within a universal closure with respect to certain free type variables - see Specification 3.2.1) it may be best to think of Proposition 2.3.5 as representing yet another modification of realizability for a special class of formulae.

\section{Program Derivation from Proofs of Specifications}

The example in this section illustrates the application of the theory to the task of deriving programs from proofs of specifications. The example is non-trivial because the recursive structure of the program we derive (equivalently: the inductive structure of the proof of the specification) is not immediately inherited from the inductive structure of the type over which the specification is defined. The example raises some interesting issues regarding the role of intensional sets in program specification and development. The topic of non-structural recursion has recently been given some attention within the field of program development in Martin-Lof's theory of types [Nor87] but this has required the addition of a new type constructor which, like other modifications to MLTT, contradicts the principle of complete presentation which is central to Martin-Lof's approach. We will begin, in Section 3.1 by setting up the infrastructure necessary for the development of this example in Section 3.2.

\subsection{Lists, Trees and Functions in TK}

We shall begin by defining a number of basic type constructors and relations which we shall need in the sequel. Elementary comprehension supports the definition of expected data types, like products, sums and functions.

$$
\begin{aligned}
X \otimes Y & =\{z \mid\langle(\text { fst } z),(\text { snd } z)\rangle=z \wedge(\text { fst } z) \in X \wedge(\text { snd } z) \in Y\} \\
X \oplus Y & =\{z \mid(\text { fst } z) \rightarrow(\text { snd } z) \in X,(\text { snd } z) \in Y\} \\
X \cup Y & =\{z \mid z \in X \vee z \in Y\} \\
X \Rightarrow_{P} Y & =\{z \mid(\forall x \in X)((z x) \downarrow \Rightarrow(z x) \in Y)\} \\
X \Rightarrow_{T} Y & =\{z \mid(\forall x \in X)((z x) \downarrow \wedge(z x) \in Y)\} \\
\left\{c_{1}, \ldots, c_{n}\right\} & =\left\{z \mid z=c_{1} \vee \ldots \vee z=c_{n}\right\} \\
\text { Bool } & =\{\text { true, false }\} \\
X \subseteq Y \Leftrightarrow(\forall x \in X)(x \in Y) & \Leftrightarrow Y \subseteq Y \subseteq X \\
X \equiv Y & \Leftrightarrow X \subseteq Y \wedge Y \subseteq X
\end{aligned}
$$

A predicate which asserts equality to a constant is decidable and so enumeration types can be reformulated in the obvious way as a negative set. Above and beyond 
these elementary constructions we can introduce polymorphisms and data abstractions.

$$
\begin{aligned}
& \Pi(X, Y)=\{z \mid(\forall X)(z \in Y)\} \\
& \Sigma(X, Y)=\{z \mid(\exists X)(z \in Y)\}
\end{aligned}
$$

$\Pi(X, Y)$ is a collection of objects which have the property of belonging to all instantiations of some type scheme ( $Y$, in interesting cases, is instantiated with an expression with $X$ free) it therefore contains what are usually understood as polymorphic objects. $\Sigma(X, Y)$, on the other hand, contains elements which belong to some instantiation of the type scheme $Y . \Sigma(X, Y)$ is a data abstraction every member of which belongs to a type formed from $Y$ with respect to an implementing type $X$. Note that these are kinds as the comprehension formulae utilise bound variables over types. Indeed, it is useful to think of $\Sigma(X, Y)$ as a universe of types of a particular sort.

It is possible to define collections of lists and $s$-expression trees in TK via inductive generation. We begin with two simple assertions:

$$
\begin{aligned}
& \psi(x, y) \Leftrightarrow(\exists a \in A)(x=\langle a, y\rangle) \\
& \beta(x, y, z) \Leftrightarrow x=\langle y, z\rangle
\end{aligned}
$$

and to use these as the basis for the following inductive types:

$$
\begin{aligned}
& \text { List }=\Xi(\psi,\{[]\}) \\
& \operatorname{Sexp}=\Xi(\beta, A)
\end{aligned}
$$

These are, lists (of elements of $A$ ) and $s$-expressions (over the set $A$ ). The token [] is just a constant of the term language. Is the set List a negative set (providing that $\boldsymbol{A}$ is)? Indeed it is, because the generating formula, $\psi$, is equivalent to $\langle($ fst $x),(\operatorname{snd} x)\rangle=x \wedge($ fst $x) \in A \wedge($ snd $x)=y$. This is a simple example of a common situation where the most perspicuous form of presentation seems to place a definition outside the important class of negative sets.

In fact it might be more natural to define lists and $s$-expressions as operations which yield types given some base type:

List $=\lambda X \Xi \Xi(\psi,\{[]\})$ where: $\psi(X, x, y) \Leftrightarrow(\exists z \in X)(x=\langle z, y\rangle)$

$\operatorname{Sexp}=\lambda X . \Xi(\beta, X)$

We will only ever use these operators over decidable sets.

In each case we obtain from the rules for $\Xi$-closure, the rule $\Xi$-Elim, and the associated combinator irec, the following introduction rules, induction principles and recursion combinators for $\operatorname{List}(A)$ and $\operatorname{Sexp}(A)$.

$$
\begin{aligned}
& \frac{a \in A \quad l \in \operatorname{List}(A)}{[] \in \operatorname{List}(A)} \text { (i) } \quad \frac{a \in}{a: l \in \operatorname{List}(A)} \text { (ii) } \\
& \frac{\psi([])(\forall x \in \operatorname{List}(A))(\forall a \in A)(\psi(x) \Rightarrow \psi(a: x))}{(\forall x \in \operatorname{List}(A))(\psi(x))}
\end{aligned}
$$

Irec $x y[]=x$

$\operatorname{lrec} x y(a: l)=y(a: l)(\operatorname{Irec} x y l)$

For $s$-expressions we obtain:

$$
\frac{a \in A}{a \in \operatorname{Sexp}(A)} \text { (i) } \quad \frac{s \in \operatorname{Sexp}(A) s^{\prime} \in \operatorname{Sexp}(A)}{s:: s^{\prime} \in \operatorname{Sexp}(A)} \text { (ii) }
$$




$$
\begin{aligned}
& \frac{(\forall x \in A)(\psi(x))(\forall x, y \in \operatorname{Sexp}(A))(\psi(x) \wedge \psi(y) \Rightarrow \psi(x:: y))}{(\forall x \in \operatorname{Sexp}(A))(\psi(x))} \\
& \operatorname{srec} x y a=x a \\
& \operatorname{srec} x y\left(s_{1}:: s_{2}\right)=y\left(s_{1}:: s_{2}\right)\left(\operatorname{srec} x y s_{1}\right)\left(\operatorname{srec} x y s_{2}\right)
\end{aligned}
$$

We have, for convenience, written the pair $\langle a, l\rangle$ as $a: l$ in the case of lists and the pair $\left\langle s_{1}, s_{2}\right\rangle$ as $s_{1}:: s_{2}$ in the case of $s$-expressions. Note how the type parameter $A$ (in both cases) does not appear explicitly in the definition of either combinator. This is a consequence of the modified realizability of bounded quantification. An immediate application for the modified induction principles establishes that $\operatorname{List}(A)$ and $\operatorname{Sexp}(A)$ satisfy the expected equations.

\section{Proposition 3.1.1}

(i) $\operatorname{List}(A) \equiv\{[]\} \cup A \otimes \operatorname{List}(A)$

(ii) $\operatorname{Sexp}(A) \equiv A \cup \operatorname{Sexp}(A) \otimes \operatorname{Sexp}(A)$

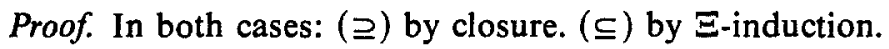

\subsection{Rotating Binary Trees - an Example Derivation}

The task we set ourselves is to derive a program which, given an arbitrary $s$-expression will rotate it into left linear form. In order to state the specification we will need two predicates. The first is is-left-linear and the second is the binary predicate eq-fringe.

$$
\begin{aligned}
& \text { is-left-linear }\left(s_{1}:: s_{2}\right)=\left(\text { atom } s_{1}\right) \&\left(\text { is-left-linear } s_{2}\right) \\
& \text { is-left-linear } a \quad=\text { true } \\
& \text { eq-fringe } \left.s_{1} s_{2}=\text { equal (fringe } s_{1}\right)\left(\text { fringe } s_{2}\right. \text { ) }
\end{aligned}
$$

where fringe is the well-known map of type: $\Pi\left(X, \operatorname{Sexp}(X) \Rightarrow_{T} \operatorname{List}(X)\right)$.

\section{Specification 3.2.1}

$$
(\forall A)(\forall x \in \operatorname{Sexp}(A))(\exists y \in \operatorname{Sexp}(A))((\text { is-left-linear } y) \wedge(\text { eq-fringe } x y))
$$

We have used an important convention here: terms with co-domain Bool are often written as if they were assertions. We suppress the equality assertion which would remove the abuse of notation. This convention corresponds, symmetrically, to the abuse we allow for conditional assertions as indicated in Section 2.3.

How should we go about proving such a specification? To begin with we can let $A$ be arbitrary and show that: $(\forall x \in \operatorname{Sexp}(A))(\exists y \in \operatorname{Sexp}(A))$ ((is-left-linear $y) \wedge($ eq-fringe $x y))$. The obvious way to proceed now is by induction on $s$-expressions using the principle we obtained in Section 3.1. This is trivial if we equip ourselves with a function join (over $s$-expressions) as follows:

$$
\begin{aligned}
& \text { join }\left(s_{1}:: s_{2}\right) s=s_{1}::\left(\text { join } s_{2} s\right) \\
& \text { join } a s=a:: s
\end{aligned}
$$

It is an easy exercise to check that (join $s_{1} s_{2}$ ) is left linear whenever $s_{1}$ and $s_{2}$ are, and furthermore that fringe (join $\left.s_{1} s_{2}\right)=$ append(fringe $s_{1}$ )(fringe $s_{2}$ ). 
Now the induction is trivial: in the base case we can take $y=x$. For the induction case we may assume ex hypothesi that $y_{1}$ and $y_{2}$ are the witnesses for $s_{1}$ and $s_{2}$ whenever $x=\left(s_{1}:: s_{2}\right)$. But then in view of the properties of join we see that we can take $y=$ (join $y_{1} y_{2}$ ) as the witness for $x$. This proof yields the following program:

$$
\begin{aligned}
& \text { rotate }\left(s_{1}:: s_{2}\right)=\text { join }\left(\text { rotate } s_{1}\right)\left(\text { rotate } s_{2}\right) \\
& \text { rotate } a=a
\end{aligned}
$$

However, this brute force solution is rather a poor program as a simple complexity analysis highlights: The number of data constructions required to form the left linear tree is quadratic in the worst case and $n \cdot \log (n)$ in the average case. Are there other, better, solutions which the theory supports? We now turn to one such derivation which results in a program which is linear in the number of data constructions required in all cases.

Suppose we had a type $\operatorname{Sexp}^{\prime}(A)$ such that $\operatorname{Sexp}(A) \equiv \operatorname{Sexp}^{\prime}(A)$. We could then prove the specification

$$
\left(\forall x \in \operatorname{Sexp}^{\prime}(A)\right)(\exists y \in \operatorname{Sexp}(A))((\text { is-left-linear } y) \wedge(\text { eq-fringe } x y)) \text {. }
$$

We shall be guided by the definition of is-left-linear in the construction of the type $\operatorname{Sexp}^{\prime}(A)$. Essentially we define $\operatorname{Sexp}^{\prime}(A)$ by constructing the characteristic subset of is-left-linear and then extending this to the entire type $\operatorname{Sexp}(A)$. This locates a creative step but it looks as if there may be principles at work here which await discovery. We shall return to this issue in Section 5.

$$
\begin{aligned}
& \operatorname{Sexp}^{\prime}=\lambda X . \Xi(\xi, X) \quad \text { where: } \\
& \xi(x, y, z) \Leftrightarrow y \in X \rightarrow x=y:: z \text {, let } u:: v=z \text { in } x=(y:: u):: v
\end{aligned}
$$

This definition yields the following introduction (closure) and elimination (induction) rules:

$$
\begin{aligned}
& \frac{a \in A}{a \in \operatorname{Sexp}^{\prime}(A)} \text { (i) } \quad \frac{a \in A s \in \operatorname{Sexp}^{\prime}(A)}{a:: s \in \operatorname{Sexp}^{\prime}(A)}(\text { ii) }) \quad \frac{x::(y:: z) \in \operatorname{Sexp}^{\prime}(A)}{(x:: y):: z \in \operatorname{Sexp}^{\prime}(A)}(\text { iii }) \\
& (\forall x \in A)(\psi(x))\left(\forall x \in \operatorname{Sexp}^{\prime}(A)\right)(\forall a \in A)(\psi(x) \Rightarrow \psi(a:: y)) \\
& (\forall x \in \operatorname{Sexp}(A))(\operatorname{let} u::(v:: w)=x \text { in } \psi(x) \Rightarrow \psi((u:: v):: w) \\
& \left(\forall x \in \operatorname{Sexp}^{\prime}(A)\right)(\psi(x))
\end{aligned}
$$

and the following recursion combinator:

$$
\begin{aligned}
& \operatorname{srec}^{\prime} x y z((u:: v):: w)=z((u:: v):: w)\left(\operatorname{srec}^{\prime} x y z(u::(v:: w))\right) \\
& \operatorname{srec}^{\prime} x y z(a:: s) \quad=y(a:: s)\left(\operatorname{srec}^{\prime} x y z s\right) \\
& \operatorname{srec}^{\prime} x y z a=x a
\end{aligned}
$$

We now wish to establish that $\operatorname{Sexp}(A) \equiv \operatorname{Sexp}^{\prime}(A)$. The following rule (which is so useful we call it "rule $\theta$ ") is used for this.

\section{Proposition 3.2.2}

The following rule is sound:

$$
\left.\frac{(\forall x)((\forall y)(\Phi(x, y) \Rightarrow y \in T) \Rightarrow x \in T) A \subseteq T}{\Xi(\Phi, A) \subseteq T} \quad \text { (rule } \theta\right)
$$

Proof. We use $\Xi(\Phi, A)$-induction. 
Base case $x \in A$. Trivial, by $\Xi$-closure and the second premise.

Induction case $x \in \mathrm{Clo}(\Xi(\Phi, A))$. We may assume $(\forall y)(\Phi(x, y) \Rightarrow y \in T)$ ex hypothesi; but the first premise provides us with $(\forall y)(\Phi(x, y) \Rightarrow y \in T) \Rightarrow x \in T$ hence $x \in T$ as required.

\section{Proposition 3.2.3}

$$
\operatorname{Sexp}(A) \subseteq \operatorname{Sexp}^{\prime}(A)
$$

Proof. By rule $\theta$ it is sufficient to show that: $\left(\forall x \in \operatorname{Sexp}^{\prime}(A)\right)\left(\forall y \in \operatorname{Sexp}^{\prime}(A)\right)$ $\left((x:: y) \in \operatorname{Sexp}^{\prime}(A)\right.$ and this we tackle by $\operatorname{Sexp}^{\prime}(A)$-induction.

Base case $x \in A$. We need to show that for any $y \in \operatorname{Sexp}^{\prime}(A)$ that $x:: y \operatorname{Sexp}^{\prime}(A)$ but this is just $\operatorname{Sexp}^{\prime}(A)$-closure(ii).

Induction case 1. Suppose that $a \in A$ and $s \in \operatorname{Sexp}^{\prime}(A)$. Let $s^{\prime} \in \operatorname{Sexp}^{\prime}(A)$ too. We have to show that $(a:: s):: s^{\prime} \in \operatorname{Sexp}^{\prime}(A)$. By $\operatorname{Sexp}^{\prime}(A)$-closure(iii) this reduces to showing that $a::\left(s:: s^{\prime}\right) \in \operatorname{Sexp}^{\prime}(A)$. This further reduces to showing that $s:: s^{\prime} \in \operatorname{Sexp}^{\prime}(A)$ by $\operatorname{Sexp}^{\prime}(A)$-closure(ii) since $a \in A$. But $s:: s^{\prime} \in \operatorname{Sexp}^{\prime}(A)$ ex hypothesi.

Induction case 2. Suppose that $u::(v:: w) \in \operatorname{Sexp}^{\prime}(A)$. We have to show that for arbitrary $s \in \operatorname{Sexp}^{\prime}(A)((u:: v):: w):: s \in \operatorname{Sexp}^{\prime}(A)$. By $\operatorname{Sexp}^{\prime}(A)$-closure(iii) this amounts to $(u::(v:: w)):: s \in \operatorname{Sexp}^{\prime}(A)$, but this follows ex hypothesi.

\section{Proposition 3.2.4}

\section{$\operatorname{Sexp}^{\prime}(A) \subseteq \operatorname{Sexp}(A)$}

Proof. By rule $\theta$ it is sufficient to show that: $(\forall y \in \operatorname{Sexp}(A))(\forall x)$ $(((\exists a \in A)(x=a:: y) \vee$ let $u::(v:: w)=y$ in $x=(u:: v):: w)) \Rightarrow x \in S)$. Let $y$ be arbitrary element of $\operatorname{Sexp}(A)$. Let $x$ be arbitrary. Assume the disjunction. Suppose that $(\exists a \in A)(x=a:: y)$ then by $\operatorname{Sexp}(A)$-closure(ii) $x \in S$. Suppose that $y=$ $u::(v:: w)$ for some $u, v, w$. Since $y \in \operatorname{Sexp}(A)$ we conclude that $u, v, w \in \operatorname{Sexp}(A)$ and then $x \in S$ by $\operatorname{Sexp}(A)$-closure(ii) (twice).

\section{Corollary 3.2.5}

$$
\operatorname{Sexp}(A) \equiv \operatorname{Sexp}^{\prime}(A)
$$

We may now prove the specification for rotating trees.

\section{Proposition 3.2.6}

$$
(\forall A)(\forall x \in \operatorname{Sexp}(A))(\exists y \in \operatorname{Sexp}(A))((\text { is-left-linear } y) \wedge \text { (eq-fringe } x y))
$$

Proof. Let $A$ be arbitrary. Since $\operatorname{Sexp}(A) \equiv \operatorname{Sexp}^{\prime}(A)$ it is sufficient to show that: $\left(\forall x \in \operatorname{Sexp}^{\prime}(A)\right)(\exists y \in \operatorname{Sexp}(A))(($ is-left-linear $y) \wedge($ eq-fringe $x y))$. This we tackle by $\operatorname{Sexp}^{\prime}(A)$-induction.

Base case. Note that $a \in \operatorname{Sexp}(A)$ by $\operatorname{Sexp}(A)$-closure and (is-left-linear $a$ ) and (eq-fringe $a a$ ) by elementary properties of the predicates is-left-linear and eq-fringe. From this we obtain $(\exists y \in \operatorname{Sexp}(A))(($ is-left-linear $y) \wedge($ eq-fringe $a y))$ by (bounded) $\exists$-introduction. But $a$ is an arbitrary element of $A$ so by (bounded) $\forall$-introduction we have $(\forall a \in A)(\exists s \in \operatorname{Sexp}(A))$ (is-left-linear $s) \wedge($ (eq-fringe $a s)$ as required. 
Induction case 1. We have to show that $(\exists y \in \operatorname{Sexp}(A))$ ((is-left-linear $y) \wedge$ (eq-fringe $(a:: s) y)$ ) when $a \in A$ and (ex hypothesi) ( $\exists y \in$ $\operatorname{Sexp}(A))(($ is-left-linear $y) \wedge($ eq-fringe $s y))$. Let us assume that $z$ satisfies (is-leftlinear $z) \wedge($ eq-fringe $s z)$ ). Now (eq-fringe $(a:: \dot{s})(a:: z))$ holds and so does (is-leftlinear $(a:: z))$, by simple properties of the predicates, so taking $y$ to be $a:: z$ we use $\exists$-introduction to obtain: $(\exists y \in \operatorname{Sexp}(A))(($ is-left-linear $y) \wedge$ (eq-fringe $(a:: s) y)$ ). The assumption regarding $z$ is now discharged by $\exists$-elimination.

Induction case 2. We have to show: $(\exists y \in \operatorname{Sexp}(A))(($ is-left-linear $y) \wedge$ (eq-fringe $((u:: v):: w) y))$ when $u, v, w \in \operatorname{Sexp}(A)$ and (ex hypothesi) $(\exists y \in$ $\operatorname{Sexp}(A))(($ is-left-linear $y) \wedge($ eq-fringe $(u::(v:: w)) y))$. Let us assume that $z$ satisfies (is-left-linear $z$ ) and (eq-fringe $(u::(v:: w)) z$ ). By simple properties of the predicates we see that (eq-fringe $(u::(v:: w))((u:: v):: w))$ so taking $y$ to be $z$ we use $\exists$-introduction to obtain: $(\exists y \in \operatorname{Sexp}(A))$ ((is-left-linear $y) \wedge($ eq-fringe $((u:: v):: w) y))$. The assumption regarding $z$ is now discharged by $\exists$-elimination.

From the proof of this proposition we can extract the following program:

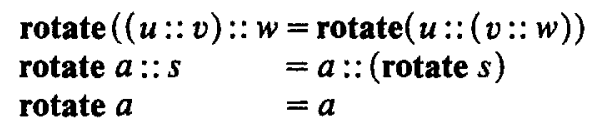

Four comments are worthwhile: rotate is an instance of the combinator srec' associated with the $\operatorname{Sexp}^{\prime}(A)$-elimination rule, which is the induction principle we have used in the proof. Secondly, it is apparent that the type $A$ does not appear in the final program. This occurs because of the definition of realizability for higher order quantification. If we write the original specification as $(\forall A) \chi$ then we see that if $e \rho(\forall A) \chi$ then $(\forall A)(e \rho \chi)$. Thirdly, we might notice that the technique we have used, that of presenting some set of elements (in this case the $s$-expressions) in a different fashion is quite reminiscent of the technique of exploring possible implementations of an abstract data type. We will return to follow up this observation in Section 5. Finally, the proof we have provided is somewhat more explicit than might be usual for what is, from a purely mathematical standpoint, a straightforward result. Naturally, in order to obtain the program the proof needs to be explicit. This tension between the informality of what DeBruijn has called the "mathematical vernacular" [Deb87] and the formality of arguments in the foundational system has perhaps never been more apparent and critical as it is in the enterprise of rigorous program development. It is not just the tension between clear and concise presentation versus explicit calculation that is at stake here because the "vernacular" has a claim to be the natural language of mathematical discourse. Some discussion of the issues of "adequacy" and "accordance" of mathematical theories appears in [Fef79] but we expect an explosion of interest in the interface between formal systems and vernacular mathematics to occur, not least because of the importance of this topic in the context of rigorous program development.

\section{Program Derivation by Transformation}

In this section we turn to the techniques of transformational programming. We feel that a theory for program development must support the further development 
of programs which have been extracted from proofs. In fact we should like to think of program extraction from proofs as a transformational step among others. Indeed, transformational program development in the spirit of [BuD76], [Bir84] and [Hen88] is another paradigm in which programming and verification are two aspects of a single strategy. Although the last point has been long understood, it is much more comprehensible within an intensional theory, and it is the aim of the current section to explore this.

Put simply, the transformational programmers maxim has been that a program transformation is an inductive proof in another guise. What the transformational programmer calls a "eureka step" a mathematician would call an induction hypothesis; what the transformational program refers to as a "fold step" the mathematician would call an appeal "ex hypothesi". The slightly unsatisfactory aspect to this is that, whilst the inductive proof referred to above is an equivalence proof between the initial and final program forms, we have to face the fact that the "proof" and the final program are developed simultaneously in the transformation. So it is rather difficult to see what proposition the program transformation is proving a priori. We shall be able to clear up this matter when we view transformation within the theory.

We shall begin with an elementary transformational technique: adding an accumulator. Our second example, which, like the example in Section 3.2, involves the reconstruction of an inductive type, concerns the technique of argument generalisation. This second example is very closely related to the example of Section 3.2 demonstrating the variety of options for development that can be accommodated by the theory.

\subsection{Adding Accumulators}

Consider the following function:

$$
\begin{aligned}
& \text { length }[]=0 \\
& \text { length }(a: l)=1+(\text { length } l)
\end{aligned}
$$

The technique of accumulator introduction is to define a new function in which to accumulate the partial resuits. Thus, conventionally we would write:

$$
\text { f } l n=(\text { length } l)+n
$$

Rather we write a specification of the following form

\section{Specification 4.1.1}

$$
(\forall A)(\forall x \in \operatorname{List}(A))(\forall z \in \mathrm{Nat})(\exists y \in \mathrm{Nat})(y=(\text { length } x)+z)
$$

Proof. There is an immediate proof of this specification from which the function $f$, above, may be extracted. However there is an alternative approach; we use $\operatorname{List}(A)$-induction.

Base step $x=[$ ]. Let $z$ be an arbitrary element of Nat. $y=($ length [ ] $)+z=z$. 
Induction step $x=(a: I) ; a \in A, l \in \operatorname{List}(A)$. Let $z$ be an arbitrary element of Nat. We have to produce some $y=($ length $(a: l))+z$. Now (length $(a: l))+z=$ (length $l)+1+z$. Our assumption is: $(\forall z \in N a t)(\exists y \in N a t)(y=($ length $l)+z)$. So we set $y=y_{1}$ where $y_{1}$ is the witness for: $(\exists y \in$ Nat $)(y=($ length $v)+z+1)$.

This proof leads to the expected equations:

$$
\begin{aligned}
& \mathbf{f}[] n=n \\
& \mathbf{f}(a: l) n=\mathbf{f} l(n+1)
\end{aligned}
$$

Although a rather simple example, this forcefully demonstrates the relationship between the structure of the proof and that of the extracted program: notice that the intensional structure of the program arises as a consequence of the intensional structure of the proof that the specification is satisfiable. In this example the elimination rule for lists also has the effect of eliminating the function length in the extracted program.

\subsection{Generalised Arguments}

Another well known and useful program development tactic is the technique of argument generalisation. We shall investigate this with respect to the following program:

$$
\begin{array}{ll}
\text { fringe }(u:: v) & =(\text { fringe } u) *(\text { fringe } v) \\
\text { fringe } a & =[a]
\end{array}
$$

where $*$ is the list-append operator of type:

$$
\Pi\left(X, \operatorname{List}(X) \Rightarrow_{T}\left(\operatorname{List}(X) \Rightarrow_{T} \operatorname{List}(X)\right)\right)
$$

The technique of generalisation suggests taking a program of type $X \Rightarrow_{r} Y$ and introducing one of type $Z \Rightarrow_{T} Y$ where there is an embedding from $X \Rightarrow_{T} Z$. In the current example we shall take $X=\operatorname{Sexp}(A)$ and $Z=\operatorname{List}(\operatorname{Sexp}(A))$ so the embedding is the obvious injection which takes each $s$-expression to a singleton list. Our required program is to form the fringe list of a sequence of $s$-expressions provided as a list. We are led to specify this as follows:

\section{Specification 4.2.1}

$(\forall A)(\forall x \in \operatorname{List}(\operatorname{Sexp}(A)))(\exists y \in \operatorname{List}(A))(y=(\operatorname{Irec}[] \operatorname{g} x))$ where $\mathbf{g}(s: I) z=($ fringe $s) * z$

Proof. As in Section 4.1, there is a very simply proof of this: Let $A$ and $x$ be arbitrary and take $y$ as indicated. This leads to the program:

$$
\mathbf{h} l=\operatorname{lrec}[] \mathrm{g} l
$$

which, as before, is the eureka definition a standard program transformation might begin with. 
The obvious approach is to attempt a $\operatorname{List}(A)$-induction but this is blocked by the definition of fringe. Rather we adopt the technique we introduced in Section 3.2, which is to find an inductive reconstruction of the type in question. Here we wish to form a type $\Xi(\delta, B)$ so that $\Xi(\delta, B) \equiv \operatorname{List}(\operatorname{Sexp}(A))$. This is a creative step, except that we may be guided by the structure of $\operatorname{List}(\operatorname{Sexp}(A))$. We ensure that we generate $\operatorname{List}(A)$ and then close off these list elements under the construction for s-expressions:

$$
\begin{aligned}
& \delta(x, y) \Leftrightarrow(\text { fst } x) \in A \rightarrow y=(\text { snd } x), \text { let } u:(v: w)=y \text { in } x=(u:: v): w \\
& B=\{[]\}
\end{aligned}
$$

This should be reminiscent of the example of Section 3.2.

\section{Proposition 4.2.2}

$$
\operatorname{List}(\operatorname{Sexp}(A)) \subseteq \Xi(\delta, B)
$$

Proof. By rule $\theta$ it is sufficient to show that $z \in \operatorname{Sexp}(A) \wedge y \in \Xi(\delta, B) \Rightarrow z: y \in$ $\Xi(\delta, B)$. This can be shown by $\operatorname{Sexp}(A)$-induction.

Base case. Trivial by $\Xi(\delta, B)$-closure.

Induction case $z=u:: v$. We have to show that $(u:: v): y \in \Xi(\delta, B)$ and this follows by $\Xi(\delta, B)$-closure providing that $u:(v: y) \in \Xi(\delta, B)$. This follows $e x$ hypothesi (twice).

\section{Proposition 4.2.3}

$$
\Xi(\delta, B) \subseteq \operatorname{List}(\operatorname{Sexp}(A))
$$

Proof. By $\Xi(\delta, B)$-induction.

Base step. Trivial.

Induction step. We may assume that $(\forall y)(\delta(x, y) \Rightarrow y \in \operatorname{List}(\operatorname{Sexp}(A)))$. Now we may conclude that $x \in \operatorname{List}(\operatorname{Sexp}(A))$ if we can show that $(\forall y)(\psi(x, y) \Rightarrow y \in$ List $(\operatorname{Sexp}(A)))$. Let $y$ be arbitrary and assume that $\psi(x, y)$. It follows immediately that $(\exists s \in \operatorname{Sexp}(A))(x=s: y)$. Suppose $x=s: y$ for some $s \in \operatorname{Sexp}(A)$. There are two cases to consider: If $s \in A$ then $\delta(x, y)$ since $(\exists a \in A)(x=a: y)$ and hence $y \in \operatorname{List}(\operatorname{Sexp}(A))$ ex hypothesi. On the other hand if $s=u:: v$ then $\delta(x, u:(v: y))$ since $x=(u:: v): y$; consequently $u:(v: y) \in \operatorname{List}(\operatorname{Sexp}(A))$ ex hypothesi and hence $y \in \operatorname{List}(\operatorname{Sexp}(A))$ as required.

\section{Corollary 4.2.4}

$$
\Xi(\delta, B) \equiv \operatorname{List}(\operatorname{Sexp}(A))
$$

We can now continue the proof of Specification 4.2 .1 by $\Xi(\delta, B)$-induction. For convenience we shall split the induction step into two separate cases. 
Base case $\mathrm{x}=[\mathrm{]}$. Take $y=[]$ since $\operatorname{Irec}[\mathrm{g}[\mathrm{]}=[\mathrm{]}$.

Induction step $1 x=(a: l)$. We have to show that $(\exists y \in \operatorname{List}(A))$ $(y=\operatorname{Irec}[] \mathbf{g}(a: l))$ which by Irec, fringe and $*$ yield: $y=($ fringe $a) *(\operatorname{Irec}[] \mathbf{g} l)=$ $[a] *(\operatorname{Irec}[] \mathrm{g} l)=a:(\operatorname{Irec}[] \mathrm{g} l)$. Now we have $a y^{\prime}=(\operatorname{Irec}[] \mathrm{g} l)$ ex hypothesi. Take $y=a: y^{\prime}$.

Induction case $2 x=(u:: v): l$. We have to show that $(\exists y \in \operatorname{List}(A))$ $(y=\operatorname{Irec}[] \mathbf{g}((u:: v): l))$ which by Irec, fringe and $*$ yield:

$$
\begin{aligned}
& y=(\text { fringe }(u:: v)) *(\operatorname{Irec}[] \mathbf{g} l)=(\text { fringe } u) *(\text { fringe } v) *(\operatorname{Irec}[] \operatorname{g} l) \\
& =(\text { fringe } u) *(\operatorname{Irec}[] \mathbf{g}(v: l))=(\operatorname{Irec}[] \operatorname{g}(u:(v: l))) .
\end{aligned}
$$

We have $y^{\prime}=\left(\operatorname{Irec}[] \mathrm{g}(u:(v: l))\right.$ ex hypothesi. Take $y=y^{\prime}$.

The program which we can extract from this proof is:

$$
\begin{aligned}
& \mathbf{h}[]=[] \text { (base case) } \\
& \mathbf{h}(a: l)=a:(\mathbf{h} l) \text { (induction case 1) } \\
& \mathbf{h}((u:: v): l=\mathbf{h}(u:(v: l)) \text { (induction case } 2)
\end{aligned}
$$

which should be more than a little reminiscent of the program of Section 3.2.

\section{Conclusions and Future Work}

There are two classes of properties of programs which are important in program development. These are the extensional and intensional properties. Correctness and the varieties of program/data abstraction concern the extensional whilst algorithmic structure, particularly with regard to issues of complexity and the implementation of data types, concern the intensional. Semantically, data types have been explicated within a classical set theoretic framework and so have been extensional objects. The most stark example is probably the specification language $Z$ [Spi88] which is essentially the classical Zermelo-Fraenkel theory. It has long been appreciated that the notion of data type should include collections of programs (either directly, in the case of functional languages, or indirectly (via denotational semantics) in the case of imperative languages). When we make this identification of programs and data types we lose, in a classical framework, the intensional aspects of programs. Moreover, since a data type is characterised only by its elements, we lose the opportunity of connecting the way a program is constructed (intensionally) with the way that the data type of its domain is constructed. That this opportunity is not lost in the framework exemplified here follows because TK is an intensional theory. Indeed, this is exactly what we have done in the examples we have studied here. We may replace the domain of a specification with any intensionally distinct (though extensionally equal) data type to obtain intensionally distinct (though extensionally equal) programs which meet it. These issues are illustrated by the example derivations of Sections 3.2 and 4.2 .

In fact we can express a particularly interesting notion of data abstraction in such an intensional theory. Consider the following kind:

$$
\{z \mid z \equiv T\} \text { (where } T \text { is some type) }
$$

We may think of this as an abstract data type since it contains all "implementa- 
tions" of a given type $T$. Note that this is only meaningful within an intensional framework since such a collection is just a singleton in a classical theory of sets.

An inductive type constructor (acc-type) has recently been added to MartinLof's theory of types [Nor87]. This has been used to derive general recursions [SaM87]. Since the theory already contains the $W$-type constructor, which also constructs inductive types, we might wonder why a new constructor is required. It is necessary to show that a program derived from a proof over an inductive type is in some sense admissible as a program over the intended domain of definition just as we have shown in this paper. The principle of unicity of types which Martin-Lof adopted (every object belongs to just one type) has to be relaxed to make this possible, just as it had to be in order to accommodate sub-typing in a natural way. Thus the acc-type complements the $W$-type in the same way that subtypes complement the general sum types (which are the only subtypes which Martin-Lof himself would countenance). We take this as evidence in favour of a theory, like TK, which is more systematically based on flexible typing.

We also utilised the intensional character of the theory in our two examples of program transformation. In this case the intensional structure of the proof (as well as the type) led to intensionally distinct programs. In fact, the specifications were trivial (as propositions) since they admitted immediate proofs; but those proofs only led to what we know traditionally as eureka definitions.

Although we were quite strict with the presentation in Section 4, there seems to be no reason why program transformations along these lines should not look much different to what is conventionally accepted. The difference will be apparent in the structure and role of the underlying data types. We are currently investigating the notion of "type simulation" [Hen88] which finds an especially satisfactory role when transformation is entertained within TK.

Many of the propositions we have had to tackle have concerned the relationship between two intensional presentations of some type. There seem to be a number of interesting possibilities here concerning the "algebra" of inductive sets. For example, the following principle is sound:

$$
(\forall(\Phi) \Rightarrow \forall(\psi)) \Rightarrow \Xi(\Phi, A) \subseteq \Xi(\psi, A)
$$

On the other hand, the converse is certainly not sound. There are a number of other useful principles of a general nature, for example:

$$
\Xi(\Phi, A) \otimes \Xi(\psi, B) \equiv \Xi(\chi, A \otimes B)
$$

where $\chi$ is a lexicographic ordering based on $\Phi$ and $\psi$. This principle, for example can be used to derive Ackermann's function in TK. Finally, it is possible to define mutually inductive systems of types (in a fairly standard way) without extra apparatus. These are crucial for even modest program derivations and require further study and investigation.

\section{Acknowledgements}

I should like to thank Ray Turner, with whom the theory TK was designed, for useful discussions. The anonymous referees provided excellent advice and assistance. 


\section{References}

Abb87] Abbas, A.: Programming with Types and Rules in Martin-Lof's Theory of Types. Doctoral Thesis, Dept Computer Science, Queen Mary College, London University, 1987.

BCM89] Backhouse, R. C., Chisholm, P., Malcolm, G. and Saaman, E.: Do-It-Yourself Type Theory. Formal Aspects of Computing, 1, 19-84 (1989).

Bee85] Beeson, M.: Foundations of Constructive Mathematics. Springer Verlag, 1985.

Bir84] Bird, R. S.: The Promotion and Accumulation Strategies in Transformational Programming. ACM Transactions on Programming Languages and Systems, 6, 487-504 (1984).

BuD76] Burstall, R. and Darlington, J.: A System which Automatically Improves Programs. Acta Informatica, 11, 1-30 (1976).

Con86] Constable, R. et al.: Implementing Mathematics with the Nuprl Proof Development System, Prentice Hall, 1986.

Deb87] De Bruijn, N. G.: The Mathematical Vernacular, a Language for Mathematics with Typed Sets. Proc. Workshop on Programming Logic, Technical Report 37, pp. 1-36, Programming Methodology Group, Chalmers University of Technology, 1987.

Fef79] Feferman, S.: Constructive Theories of Functions and Classes. Logic Colloquium '78, pp. 159-224, North Holland, 1979.

HaN87] Hayashi, S and Nakano, H.: The PX System - a Computational Logic. Publications of the Research Institute for Mathematical Sciences, Kyoto University, Tokyo, 1987.

Hen87] Henson, M. C.: Elements of Functional Languages, Blackwell, 1987.

Hen88] Henson, M. C.: Higher Order Transformations and Type Simulations, Computer Journal, 31, 517-524 (1988).

HeT88] Henson, M. C. and Turner, R.: A Constructive Set Theory for Program Development. Proc. 8th Conf. on Foundations of Software Technology and Theoretical Computer Science, K. V. Nori and S. Kumar (eds) Lecture Notes in Computer Science 338, pp. 329-347, Springer Verlag, 1988.

Kha86] Khamiss, A.: Algorithm Development in Martin-Lof's Theory of Types. Doctoral Thesis, Dept Computer Science, University of Essex, 1986.

Kle45] Kleene, S.: On the Interpretation of Intuitionistic Number Theory. Journal of Symbolic Logic, 10, 109-124 (1945).

MaC88] Malcolm, G. and Chisholm, P.: Polymorphism and Information Loss in Martin-Lof's Type Theory. Technical Report CS 8814, Dept. Maths and Computing Sciences, University of Groningen, 1988.

Mar82] Martin-Lof, P.: Constructive Mathematics and Computer Programming. In: Logic, Methodology and Philosophy of Science VI, pp. 153-175, North Holland, 1982.

Moh85] Mohring, C.: Algorithm Development in the Calculus of Constructions. Proc. IEEE Symp. on Logic in Computer Science, pp. 84-91, 1986.

Nor87] Nordstrom, B.: Terminating General Recursion. Technical Report, Dept Computer Science, University of Goteborg, 1987.

Pey87] Peyton-Jones, S.: The Implementation of Functional Programming Languages, Prentice Hall, 1987.

SaM87] Saaman, E. and Malcolm, G.: Well-Founded Recursion in Type Theory. Technical Report, Subfaculteit Wiskunde en Informatica, Rijksuniversiteit Gronigen, 1988.

Spi88] Spivey, J. M.: Understanding Z, Cambridge University Press, 1988.

Tur79] Turner, D. A.: A New Implementation Technique for Applicative Languages. Software, Practice and Experience, 9, 31-49 (1979).

Received September 1988

lccepted in a revised form in February 1989 by D. Simpson 